\title{
DEPENDENCE OF PANCREAS FUNCTIONAL CAPACITY AT CHRONIC PANCREATITIS ON ENDOTOXICOSIS AND OTHER METABOLIC FACTORS
}

DOI: 10.36740/WLek202104110

\author{
Liliya S. Babinets, Olexandr S. Zemlyak, Iryna M. Halabitska, Halyna M. Sasyk, Zoreslava Ya. Onufryk \\ HORBACHEVSKY TERNOPIL NATIONAL MEDICAL UNIVERSITY, TERNOPIL, UKRAINE
}

\begin{abstract}
The aim: Was to investigate the status of endotoxicosis parameters in patients with chronic pancreatitis depending on the presence of a combination with type 2 diabetes mellitus, as well as their impact on the functional capacity of the pancreas.

Materials and methods: 87 outpatients with CP with concomitant type 2 diabetes and without it were examined. The main group consisted of 62 patients with $C P$ in the phase of therapeutic exacerbation in combination with diabetes mellitus in a state of sub- or full compensation, the comparison group - 25 patients with isolated CP, the control group consisted of 30 healthy individuals. The content of malonic aldehyde in the blood was determined by reaction with thiobarbituric acid, the levels of medium-molecular peptides MMP1 and MMP2 - by the method of Gabrielyan, circulating immune complexes - by precipitation in $3.75 \%$ ethylene glycol with followed photometry.

Results: The presence of active endotoxicosis and lipid peroxidation in CP was established, which was significantly more significant in the comorbidity of CP with type 2 diabetes: erythrocyte intoxication index was higher by $19.2 \%$, the content of medium molecules MMP1 - by $29.5 \%$, MMP2 - by $35.4 \%$, malonic aldehyde - $10.9 \%$, circulating immune complexes $-23.9 \%$, ceruloplasmin - by $11.9 \%(p<0.05)$

Conclusions: A deeper level of excretory and incretory insufficiency of the pancreas in concomitant diabetes mellitus 2 , which deepened with increasing endotoxicosis by the level of erythrocyte intoxication index based on an increase in the strength of significant moderate and moderate inverse correlations between it and fecal a-elastase such in isolated $C P$ (respectively $r=-0.517$ and $r=-0.471, p<0.05$ ) and significant direct moderate and medium strength correlations between levels of $H b A 1 c$ and erythrocyte intoxication index - respectively $r=0.552$ and $r=0.337, p<0.05$.
\end{abstract}

KEY WORDS: chronic pancreatitis, type 2 diabetes mellitus, endotoxicosis, medium molecular weight peptides, erythrocyte intoxication index, functional insufficiency of the pancreas

Wiad Lek. 2021;74(4):869-873

\section{INTRODUCTION}

The progression of chronic pancreatitis (CP), especially in combination with type 2 diabetes mellitus (DM2), occurs under conditions of oxidative stress and chronic inflammation due to both specific and nonspecific factors that determine the constant presence of varying degrees of endotoxicosis $[1,2]$. In CP, the activation of free radical or lipid peroxidation (LPO) processes leads to systemic membranopathy with the development of regenerative-plastic insufficiency syndrome at the level of the whole organism, which determines the multiorgans of the pathology with predominant lesions of barrier systems and detoxifying organs [3]. Aldehydes, LPO products, are able to activate mononuclear cells, including pancreatic star cells and Kupffer cells, which produce collagen, the liver, cause cross-linking of cytokeratins to form Mallory cells, and stimulate neutrophil chemotaxis. In CP and DM2, some of the free fatty acids coming from the intestine or formed in the liver are transported to the mitochondria, where $\beta$-oxidation and subsequent oxidation occur in the Krebs cycle. In the process of regeneration of reduced forms of coenzyme electrons, NAD and FAD are transferred to the mitochondrial respiratory chain, where phosphorylation of ADP occurs with the formation of ATP. It is known that even during normal operation of the respiratory chain, some electrons react with oxygen, which leads to the synthesis of reactive oxygen species (ROS) - superoxide anionic radicals, hydrogen peroxide, singlet oxygen, hydroxyl radical, nitroxyl radical, and others. [4]. The formation of ROS is enhanced under the influence of factors that disrupt the respiratory chain - these are endogenous and exogenous substances such as TNF- $\alpha$, medium molecular weight peptides (MMP), nitric oxide, circulating immune complexes (CIC), iron, copper, ethanol, cerulop, drugs, etc. It has long been proven that long-term or excessive intake of ethanol, which is one of the etiological factors of $\mathrm{CP}$, leads to the synthesis of cytochrome P450 2E1, the main enzyme of the microsomal system of ethanol oxidation, and increased ROS formation [5]. Separation of oxidation and phosphorylation leads to the formation of excess ROS, which, in turn, cause damage to mitochondrial DNA, which exacerbates dysfunction of the respiratory chain. 
ROSs have direct cytotoxicity by activating Fas-mediated apoptosis of pancreatic cells. ROSs also initiate LPO, exacerbating endotoxicosis, leading to the formation of toxic compounds, including malonic aldehyde (MA), 4-hydroxynonenal F-isoprostanes, etc., which can both directly cause the death of pancreatic cells and hepatocytes, and induce cytotoxic immune reactions due to covalent binding to cellular proteins, as well as the formation of cytokeratins (Mallory cells), fibrogenesis and chemotaxis of polymorphonuclear neutrophils. In addition, ROS promote the secretion of cytokines (TNF- $\alpha$, TGF- $\beta$, IL- 8 ) by mononuclear phagocytes, which potentiate these pathological processes. It is established that the increase of LPO in CP contributes to changes in the morpho-functional properties of erythrocytes: their ability to deformation and resistance to LPO decreases, catecholamine-depositing function changes, which deepens hypoxia. Thus, the vicious circle of $\mathrm{LPO}$ and endotoxicosis is closed. It is proved that in CP and DM2 there is an increase in the level of CIC, which under certain conditions can be fixed in the vessels and cause an inflammatory reaction. In chronic processes, they circulate in the blood for a long time, maintaining the conditions for the defeat of target tissues [6]. It has been established that at low concentrations CICs can cause B-lymphocyte proliferation, and at high concentrations they can inhibit it. There are reports that under the influence of high concentrations of CIC there is an increase in the activity of T-suppressors, especially those that contain $\operatorname{IgG}$, as well as inhibition of the activity of natural killers [7]. A well-known pro-inflammatory component is ceruloplasmin, which is, on the one hand, an active antioxidant and, on the other hand, a marker of active inflammation and endotoxicosis.

All these factors, in our opinion, can contribute to the maintenance of inflammatory chronic process in the pancreas, as well as torpid destructive changes in the tissues and organs of patients with CP in combination with DM2, deepening the functional insufficiency of the pancreas, which makes further study $[8,9]$. This is important to consider in the comprehensive treatment of such comorbid patients, which would improve their quality of life, would stop the further progression of CP and diabetes through rational correction of pancreatic dysfunction [10].

\section{THE AIM}

The aim of the study was to investigate the state of the parameters of endotoxicosis in patients with chronic pancreatitis, depending on the presence of a combination with type 2 diabetes, as well as their impact on the functional capacity of the pancreas.

\section{MATERIALS AND METHODS}

We examined 87 outpatients with $\mathrm{CP}$ with concomitant DM2 and without it, who were registered at the Ternopil Center of Primary Health Care. The average age was (49.33 \pm 11.54 ) years. The duration of CP in the entire cohort of patients ranged from 1-30 years, the average duration was
$(10.05 \pm 0.83)$ years. The duration of comorbidity of $\mathrm{CP}$ and $\mathrm{DM} 2$ ranged from 0 to 25 years, the average duration was $(8.93 \pm 0.60)$ years, in $76 \%$ of patients the disease lasted up to 10 years, in the remaining $24 \%$ - more than 10 years, in $24 \%$ - up to 5 years. The diagnosis of CP was established according to the clinical and statistical classification proposed by the State Institution "Research Institute of Gastroenterology of the National Academy of Medical Sciences of Ukraine" in 2003, as well as the Order of the Ministry of Health of Ukraine dated 10.09.2014 № 638 “On approval and implementation of medical documents». The diagnosis of DM2 was verified by the Order of the Ministry of Health of Ukraine from № 1118 from 21.12.2012 “On approval and implementation of medical and technological documents for standardization of medical care for type 2 diabetes".

The main group consisted of 62 patients with $\mathrm{CP}$ in the phase of therapeutic exacerbation in combination with diabetes mellitus in a state of sub- or full compensation, the comparison group - 25 patients with isolated $\mathrm{CP}$, and the study groups were comparable in age, sex, duration and treatment of the underlying disease in the previous stages. The control group consisted of 30 healthy individuals who were representative of the study groups by age, sex and social status.

The state of LPO as a marker of oxidative stress was evaluated by the content of MA, the determination of which is based on the fact that at high temperatures in an acidic environment MA reacts with thiobarbituric acid, forming a colored trimethine complex. Evaluation of endogenous intoxication (EI) was performed by the levels of MMP1 and MMP2 by the method of Gabrielyan: studied the optical density of serum at wavelengths of 254 and $280 \mathrm{~nm}$, thus determining the content of those MMP, which contain aromatic amino acids, and those that do not contain. The CIC level was determined by selective precipitation in 3.75\% ethylene glycol, followed by photometry. The EI index (IEI) was determined by the Togaibayev method, which is based on the idea of erythrocytes as a universal adsorbent. Determination of CP content was performed taking into account its exceptional ability to oxidize $n$-phenylenediamine dihydrochloride to form a violet compound.

\section{RESULTS}

In the table 1 presents the obtained data of studying the parameters of endotoxicosis in CP depending on the presence of comorbid DM2. The analysis of the obtained results allowed to prove the presence of active EI and LPO in CP, which was significantly more significant in the comorbidity of CP with DM2: erythrocyte IEI was higher by $19.2 \%$, the content of MMP1 - by $29.5 \%$, MMP2 - by $35.4 \%$, MA - $10.9 \%$, CIC - 23.9\% ( $\mathrm{p}<0.05)$. Determination of CIC content is a cost-effective marker not only of EI, but also of strengthening the protective response of the immune system by increasing the number of antigen-antibody complexes that accumulate and damage the pancreas and other organs. 
Table I. Comparative analysis of the parameters of endotoxicosis and LPO at CP depending on the presence of concomitant DM2

\begin{tabular}{|c|c|c|c|c|c|c|}
\hline El parameter & $\begin{array}{l}\text { Control group } \\
(n=30)\end{array}$ & $\begin{array}{l}\text { Patients with CP } \\
\quad(n=25)\end{array}$ & $\begin{array}{l}\text { Patients with CP and DM2 } \\
\qquad(\mathrm{n}=62)\end{array}$ & $\mathbf{p}_{1}$ & $\mathbf{p}_{2}$ & $\mathbf{p}_{3}$ \\
\hline IEl, \% & $27,25 \pm 1,22$ & $49,23 \pm 2,65$ & $58,69 \pm 1,46$ & $<0,05$ & $<0,05$ & $<0,05$ \\
\hline MMP1 & $334,11 \pm 2,64$ & $438,71 \pm 21,10$ & $568,44 \pm 18,29$ & $<0,05$ & $<0,05$ & $<0,05$ \\
\hline MMP2 & $147,53 \pm 1,23$ & $206,26 \pm 10,86$ & $279,25 \pm 7,37$ & $<0,05$ & $<0,05$ & $<0,05$ \\
\hline $\mathrm{MA}, \mu \mathrm{mol} / \mathrm{I}$ & $2,81 \pm 0,095$ & $5,31 \pm 0,14$ & $5,89 \pm 0,11$ & $<0,05$ & $<0,05$ & $<0,05$ \\
\hline CIC, con. un. & $64,19 \pm 1,65$ & $104,29 \pm 1,34$ & $129,38 \pm 1,41$ & $<0,05$ & $<0,05$ & $<0,05$ \\
\hline ruloplasmin, mg/l & $245,60 \pm 2,61$ & $305,47 \pm 8,91$ & $341,70 \pm 9,45$ & $<0,05$ & $<0,05$ & $<0,05$ \\
\hline
\end{tabular}

Note: 1. $p_{1}$ - the reliability of the difference in the parameters of patients with CP in relation to such control groups;

2. $p_{2}$ - the reliability of the difference in the parameters of patients with $C P$ and DM2 in relation to such control groups;

3. $P_{3}$ - the significance of the difference in the parameters of patients with $C P$ and DM2 in relation to such groups with isolated $C P$.

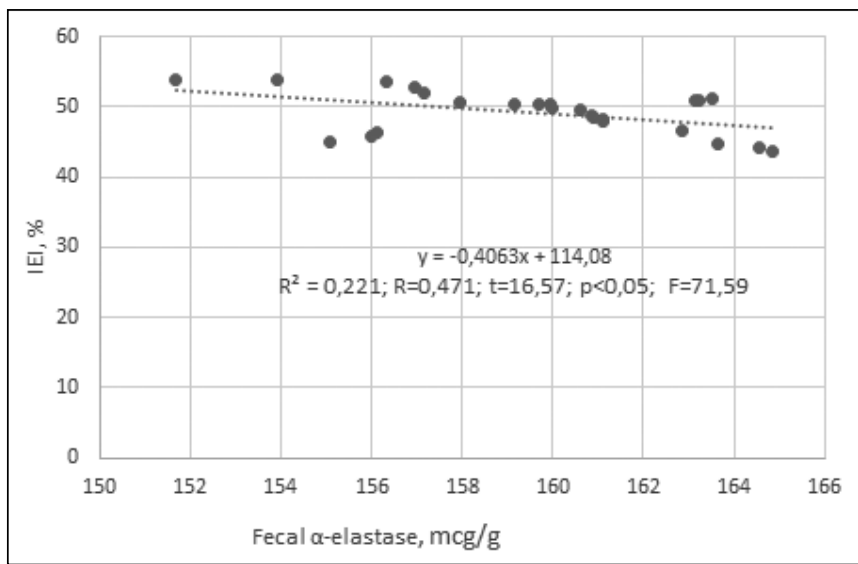

Fig. 1. Dependence of the level of fecal a-elastase on IEl, $\%$ of patients with CP

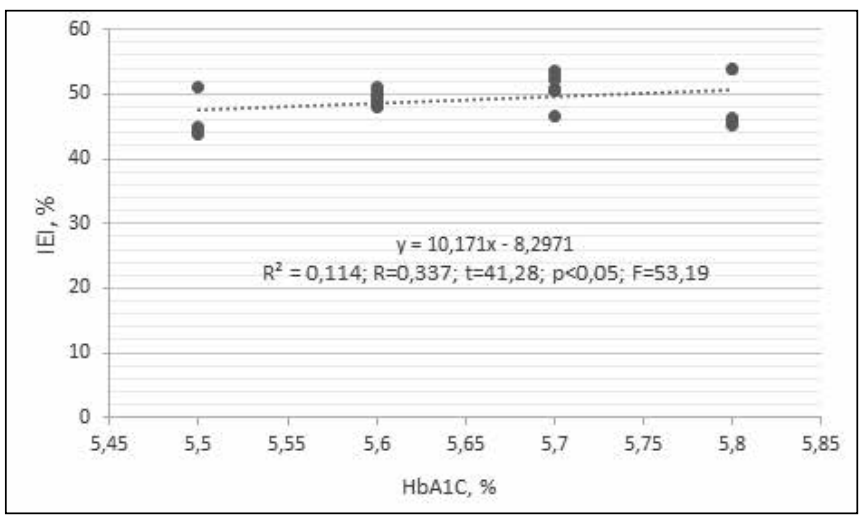

Fig. 3. Dependence of $\mathrm{HbA1C} \%$ on the level of IEl of patients with CP

We analyzed the dependence of excretory (on the level of fecal $\alpha$-elastase) and excretory (on the content of $\mathrm{HbA} 1 \mathrm{c}, \%$ ) functions of the pancreas in patients with CP and CP in comorbidity with DM2 (data in Fig. 1-2) from IEI,\%. IEI was considered one of the indicative integrative markers of endotoxicosis, so its relationship with the parameters of the functional capacity of the CP can be judged on the role of EI in the course of CP in combination with DM2.

The data of fig. 1-2 indicate a significant effect of IEI on the level of exocrine insufficiency of the pancreas in the

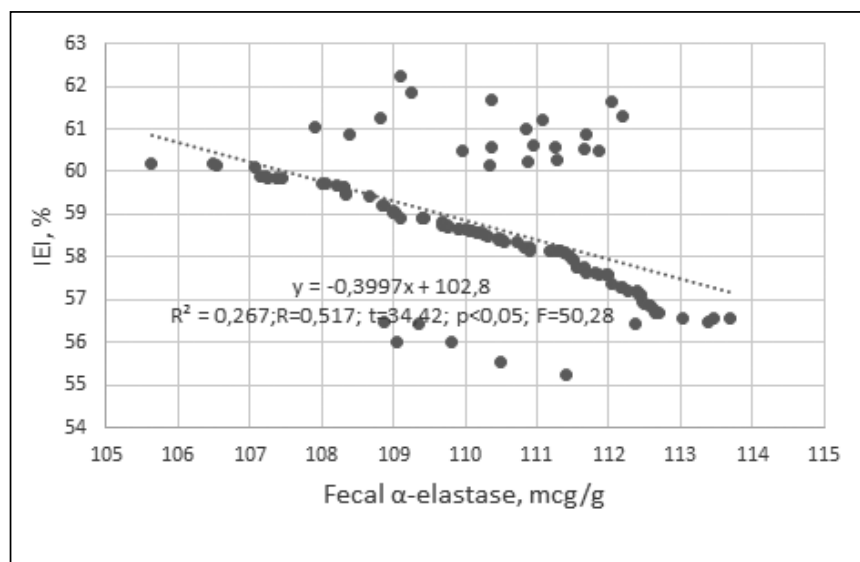

Fig. 2. Dependence of fecal a-elastase on IEl, $\%$ of patients with CP in combination with DM2

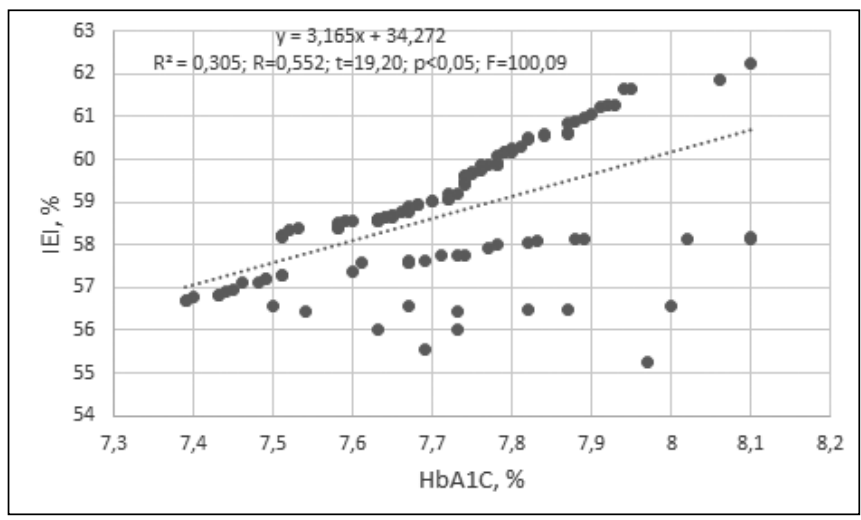

Fig. 4. Dependence of $\mathrm{HbA1C}, \%$ on the level of IEI of patients with CP in combination with DM2

content of fecal $\alpha$-elastase, which was more significant in the combined course of CP and DM2 in comparison with that at CP: this is evidenced by the established moderate and moderate inverse correlations between IEI and fecal a-elastase in patients with CP and comorbidity of CP and DM2 (respectively $\mathrm{r}=-0,471$ and $\mathrm{r}=-0,517, \mathrm{p}<0,05$ ).

According to the correlation analysis (Fig. 3-4), it was also proved to increase the strength of significant direct moderate and medium correlations between the levels of $\mathrm{HbAlc}$ and IEI in patients with isolated CP and CP 
in combination with DM2 (respectively $\mathrm{r}=0.337$ and $\mathrm{r}=0.552, \mathrm{p}<0.05$ ), which proved a deeper level of incretory insufficiency of the pancreas in concomitant DM2, which deepened with increasing endotoxicosis at the level of IEI.

$\mathrm{CP}$ activation was also found to be $11.9 \%(\mathrm{p}<0.05)$, which can be explained by the presence of certain reserves of the antioxidant defense system even in such comorbid patients. The statistically significant increase at $\mathrm{CP}$ relative to the control group and especially in concomitant DM2 relative to the group of isolated $\mathrm{CP}$ can also be explained by the activity of inflammation and endotoxicosis in the phase of mild therapeutic exacerbation of $\mathrm{CP}$, which can be performed in an outpatient setting.

\section{DISCUSSION}

Many studies have been found to investigate the effects of CP on DM2 [11, 12]. Gregory, P.K et al studied influence of non-derivative ducts of the pancreas and inflammation on the development of exocrine insufficiency of the pancreas and other trophological indicators [13]. Hollemans, R.A. et al studied the effect of changes in the pathogenetic parameters of various body systems on the development of exocrine insufficiency of the pancreas as a result of acute pancreatitis [14]. The influence of changes in the inflammatory system and other pathological changes on the development of exocrine insufficiency of the pancreas was also studied [15]. The influence of dysbiotic changes of intestinal microflora on the development and deepening of exocrine insufficiency of the pancreas has been studied [16]. The effect of exocrine insufficiency treatment on pathogenetic parameters and trophological status parameters was also studied [17]. The influence of trophological status parameters on the development of pathological processes in patients with chronic pancreatitis has been studied. [18]. However, we did not find a study that would show the influence of endotoxicosis parameters on the development of exocrine insufficiency of the pancreas and other trophological indicators.

Therefore, the obtained data proved the fact that the formation of endocrine insufficiency of the pancreas in $\mathrm{CP}$ in the form of DM2 occurs against the background of increased EI, LPO and immune disorders. The increase in the content of the pool of MMP, MA and CIC in the body with comorbidity of DM2 proves the presence of deepening of homeostasis due to increased catabolic processes and an increase in their toxic effects. In addition, the increase in the content of the CIC confirms the presence of an active inflammatory process in CP in the phase of therapeutic exacerbation. This figure was significantly higher in CP with concomitant DM2.

Thus, the obtained data proved the presence of inflammation, endotoxicosis and LPO in the long-term course of CP and comorbidity of CP and DM2, which is the cause and consequence of deepening exocrine and endocrine insufficiency of the pancreas according to the correlation analysis.

A limitation of our study is not too many indicators that we studied in the groups of patients during treatment and rehabilitation included in the study.

\section{CONCLUSIONS}

1) proved the presence of CP in the active course of endotoxicosis and lipid peroxidation, which was significantly more significant in the comorbidity of CP with DM2: erythrocyte intoxication index was higher by $19.2 \%$, the content of medium molecules MMP1 - by $29.5 \%$, MMP2 - by $35.4 \%$, malonic aldehyde - 10.9\%, circulating immune complexes $-23.9 \%$, ceruloplasmin - by $11.9 \%(\mathrm{p}<0.05)$

2) proved a deeper level of excretory and incretory insufficiency of the pancreas in concomitant diabetes mellitus, which deepened with increasing endotoxicosis by erythrocyte intoxication index based on an increase in the strength of significant moderate and moderate inverse correlations between it and fecal $\alpha$-elastase such in isolated CP (respectively $\mathrm{r}=-0.517$ and $\mathrm{r}=-0.471, \mathrm{p}<0.05)$ and significant direct moderate and medium strength correlations between levels of $\mathrm{HbAlc}$ and erythrocyte intoxication index - respectively $\mathrm{r}=0.552$ and $\mathrm{r}=0.337, \mathrm{p}<0,05$.

In the future, we plan to study the manifestations of trophological insufficiency of patients with CP and DM2 and to propose methods for adequate correction of established pathogenetic and clinical disorders.

\section{REFERENCES}

1. Babinets L.S., Halabitska I.M. Chronic inflammatory process and bone tissue changes in patients with osteoarthritis and exocrine pancreatic insufficiency Lekarsky Obzor. 2020; 69 (1): 7-10.

2. Yu J.H, Kim H. Oxidative stress and inflammatory signaling in cerulein pancreatitis. World J Gastroenterol. 2014;14;20(46): 17324-9. doi: 10.3748/wjg.v20.i46.17324.

3. Babinets L., Medvid I., Herasymets I. The imbalance of the autonomic nervous system in the patients with comorbidity of hypertonic disease and chronic pancreatitis. Lekarsky Obzor. 2014; 63(6): 211-215.

4. DiMagno M.J., DiMagno E.P.Chronic pancreatitis. Curr Opin Gastroenterol. 2012;28(5):523-31. doi: 10.1097/MOG.0b013e3283567dea.

5. Kothari D., Ketwaroo G., Freedman S.D., Sheth S.G. The Impact of risk factors of chronic pancreatitis on secretin pancreatic function testing: results of a 20-year study. Pancreas. 2017;46(7):887-890. doi: 10.1097/ MPA.0000000000000871.

6. Lohr J.M., Oliver M.R., Frulloni L. Synopsis of recent guidelines on pancreatic exocrine insufficiency. United European Gastroenterol J. 2013;I(2):79-83. doi: 10.1177/2050640613476500.

7. Kothari D., Ketwaroo G., Freedman S.D., Sheth S.G. The Impact of risk factors of chronic pancreatitis on secretin pancreatic function testing: results of a 20-year study. Pancreas. 2017;46(7):887-890. doi: 10.1097/ MPA.0000000000000871.

8. Levy P., Dominguez-Munoz E. et al. Epidemiology of chronic pancreatitis: burden of the disease and consequences. United European Gastroenterol J. 2014;2(5):345-54. doi: 10.1177/2050640614548208.

9. Yang D., Forsmark C.E. Chronic pancreatitis. Curr Opin Gastroenterol. 2017;33(5):396-403. doi: 10.1097/M0G.0000000000000377.

10. Lew D., Afghani E., Pandol S. Chronic pancreatitis: current status and challenges for prevention and treatment. Dig Dis Sci. 2017;62(7):17021712. doi: 10.1007/s10620- 017-4602-2.

11. Levy P., Dominguez-MunozE. et al. Epidemiology of chronic pancreatitis: burden of the disease and consequences. United European Gastroenterol J. 2014;2(5):345-54. 
12. Lew D., Afghani E., Pandol S. Chronic pancreatitis: current status and challengesfor prevention and treatment. Dig Dis Sci. 2017;62(7):1702-1712.

13. Gregory P.C., Hoffmann K., Kamphues J., Mobeler A. The pancreatic duct ligated (Mini)pig as a model for pancreatic exocrine insufficiency in man. Pancreas. 2016;45 (9):1213-1226. doi: 10.1097/ MPA.0000000000000674.

14. Hollemans R.A., Hallensleben N.D.L., Mager D.J. et al. Pancreatic exocrine insufficiency following acute pancreatitis: Systematic review and study level meta-analysis. Pancreatology. 2018;18 (3):253262. doi: 10.1016/j.pan.2018.02.009.

15. LindkvistB., Phillips M.E.,Domínguez-MuñozJ.E.Clinical, anthropometric and laboratory nutritional markers of pancreatic exocrine insufficiency: Prevalence and diagnostic use. Pancreatology. 2015;15 (6):589-597. doi: 10.1016/j.pan.2015.07.001.

16. Manor 0., Levy R., Pope C.E. et al. Metagenomic evidence for taxonomic dysbiosis and functional imbalance in the gastrointestinal tracts of children with cystic fibrosis Scientific Reports. 2016; 6, art. no. 22493. doi: 10.1038/srep22493.

17. Dominguez-MuñozJ.E. Management of pancreatic exocrine insufficiency Current Opinion in Gastroenterology. 2019;35(5):455-459. doi: 10.1097/ MOG.0000000000000562.

18. Stigliano S., Waldthaler A., Martinez-Moneo E., et al. Vitamins D and $K$ as Factors Associated with Osteopathy in Chronic Pancreatitis: A Prospective Multicentre Study (P-BONE Study). Clinical and Translational Gastroenterology. 2018;9 (10): 197. doi: 10.1038/s41424-018-0066-8.
Research topic: 0118U000361, Comprehensive approach to the control of symptoms, direct and distant prognosis in conditions of comorbid pathology in the clinic of internal diseases and the practice of a family medicine.

\section{ORCID and contributionship:}

Liliya S. Babinet:0000-0002-0560-1943 ${ }^{A, B, C, D, E, F}$

Olexandr S. Zemlyak: 0000-0002-6346-0931 $1^{A, B, C, D, E, F}$

Iryna M. Halabitska: 0000-0002-9028-7230 A,B,C,D,E,F

Halyna M. Sasyk: 0000-0002-9640-9155 ${ }^{A, B}$

Zoreslava Ya. Onufryk : 0000-0002-1273-7945 A,B

\section{Conflict of interest:}

The Authors declare no conflict of interest.

\section{CORRESPONDING AUTHOR Liliya S. Babinets \\ Horbachevsky Ternopil National Medical University \\ 14 Kupchyns'ky St., 46000 Ternopil, Ukraine \\ tel:+380673520743 \\ e-mail:lilyababinets@gmail.com}

Received: 21.10 .2020

Accepted: 02.03 .2021

A - Work concept and design, B - Data collection and analysis, C - Responsibility for statistical analysis,

D -Writing the article, $\mathbf{E}$ - Critical review, $\mathbf{F}$ - Final approval of the article 